\title{
Impactos do uso de bebidas alcoólicas por profissionais de enfermagem e a relação com o trabalho: uma reflexão teórica
}

Impacts of the use of alcoholic beverages by nursing professionals and the relationship with work: a theoretical reflection

Impactos del uso de bebidas alcohólicas por los profesionales de enfermería y la relación con el trabajo: una reflexión teórica

\section{Resumo}

Objetivo: Descrever os impactos do uso de bebidas alcoólicas por profissionais de enfermagem e a relação com o trabalho, a partir de uma reflexão teórica. Metodologia: Ensaio teórico de caráter reflexivo, da literatura sobre os impactos do uso de bebidas alcoólicas por profissionais de Enfermagem e a relação com o trabalho, por meio de uma revisão bibliográfica não sistemática, com abordagem qualitativa, sobre o tema "uso de bebidas alcoólicas por profissionais de enfermagem e relação com o trabalho". Para a revisão bibliográfica foi utilizado LILACS, CINAHL, BDENF, MEDLINE e Web of Science com as referidas estratégias e recorte temporal de 2010 a 2020, o levantamento limitou-se aos artigos publicados em português, inglês e espanhol. Resultados: A análise da literatura foi disposta em dois subtemas: "Impactos do uso de bebidas alcoólicas por profissionais de Enfermagem" e "Fatores associados ao consumo de bebidas alcoólicas no ambiente de trabalho da enfermagem". Conclusão: Destacaram- se os danos que repercutem diretamente na qualidade da assistência, comprometendo não só a segurança do paciente, mas ocasionando uma problematização que afeta todo o sistema institucional de saúde e referente aos fatores associados ao consumo de bebidas alcoólicas no ambiente de trabalho da enfermagem, houve destaque para os fatores estressores, para a crise sanitária atual em decorrência da Covid-19 e do isolamento social.

Palavras-chave: Bebidas Alcoólicas; Equipe de enfermagem; Saúde do trabalhador.

\section{Abstract}

Objective: To describe the impacts of the use of alcoholic beverages by nursing professionals and the relationship with work, based on a theoretical reflection. Methodology: Theoretical essay of a reflective nature, from the literature on the impacts of the use of alcoholic beverages by nursing professionals and the relationship with work, through a non-systematic bibliographic review, with a qualitative approach, on the theme "use of alcoholic beverages by nursing professionals and their relationship with work". For the literature review, LILACS, CINAHL, BDENF, MEDLINE and Web of Science were used with the referred strategies and time frame from 2010 to 2020, the survey was limited 
to articles published in Portuguese, English and Spanish. Results: The literature analysis was arranged in two subthemes: "Impacts of the use of alcoholic beverages by nursing professionals" and "Factors associated with the consumption of alcoholic beverages in the nursing work environment". Conclusion: The damages that directly affect the quality of care were highlighted, compromising not only patient safety, but causing a problem that affects the entire institutional health system and regarding factors associated with the consumption of alcoholic beverages in the workplace of In nursing, stressors were highlighted, as well as the current health crisis as a result of Covid-19 and social isolation.

Keywords: Alcoholic beverages; Nursing team; Worker's health.

\section{Resumen}

Objetivo: Describir los impactos del uso de bebidas alcohólicas por parte de los profesionales de enfermería y la relación con el trabajo, a partir de una reflexión teórica. Metodología: Ensayo teórico de carácter reflexivo, a partir de la literatura sobre los impactos del uso de bebidas alcohólicas por parte de los profesionales de enfermería y la relación con el trabajo, mediante una revisión bibliográfica no sistemática, con enfoque cualitativo, sobre el tema “consumo de bebidas alcohólicas bebidas de los profesionales de enfermería y su relación con el trabajo ". Para la revisión de la literatura se utilizaron LILACS, CINAHL, BDENF, MEDLINE y Web of Science con las estrategias referidas y el marco temporal de 2010 a 2020, la encuesta se limitó a artículos publicados en portugués, inglés y español. Resultados: El análisis de la literatura se organizó en dos subtemas: "Impactos del uso de bebidas alcohólicas por profesionales de enfermería" y "Factores asociados al consumo de bebidas alcohólicas en el ámbito laboral de enfermería". Conclusión: Se destacaron los daños que afectan directamente la calidad de la atención, comprometiendo no solo la seguridad del paciente, sino provocando un problema que afecta a todo el sistema institucional de salud y en cuanto a factores asociados al consumo de bebidas alcohólicas en el lugar de trabajo de En enfermería, se presentaron factores estresantes. destacado, así como la actual crisis sanitaria como consecuencia del Covid-19 y el aislamiento social.

Palabras-clave: Bebidas alcohólicas; Equipo de enfermeira; Salud del trabajador.

\section{Introdução}

O consumo de bebidas alcoólicas tem se configurado como um problema global, diante do aumento entre a população. Segundo a Organização Mundial da Saúde (OMS), o consumo mundial per capita de álcool puro foi de 6,4 litros em 2016, com projeções de aumento para os próximos 10 anos. Na região das Américas, o consumo per capita foi de 8 litros e, no Brasil, o valor médio foi de 7,8 litros. Quando se avaliaram somente as pessoas que fizeram uso nos 12 meses anteriores à pesquisa, $43 \%$ da população mundial estavam nesta categoria. Nas Américas, a frequência foi de 54,1\% e no Brasil, foi igual a $40 \%$. Sobre o consumo médio diário: o índice brasileiro foi de 03 doses padrão 4 (41,7 gramas/dia), enquanto nas Américas e no mundo foram de 2,3 doses - 32,8 gramas/dia (OMS, 2018).

A Organização Mundial da Saúde (OMS) destaca que o papel do álcool sobre problemas de saúde crônicos e agudos são determinados por duas dimensões de consumos distintos, mas que se relacionam entre si: o volume total consumido; e o padrão de consumo. Todavia, há dificuldade nas definições dos padrões de consumo na literatura, pois a definição do uso excessivo apresenta divergências entre e dentro dos países (OMS, 2018).

No Brasil é adotado o conceito de dose padrão, a unidade que define a quantidade de etanol puro nas bebidas alcoólicas. Assim, uma dose de bebida equivale a $14 \mathrm{~g}$ de álcool puro, o que corresponde a $350 \mathrm{ml}$ de cerveja (5\% de álcool), $150 \mathrm{ml}$ de vinho (12\% de álcool) ou $45 \mathrm{ml}$ de destilado (vodca, uísque, cachaça, gin, tequila, com 40\% de álcool) (Andrade, 2020).

Neste contexto, embora o álcool possua um caráter lícito, essa substância é psicoativa e pode gerar dependência. Na perspectiva da relação entre consumo de álcool e trabalho, destaca-se que os problemas relacionados ao seu uso podem prejudicar a saúde do trabalhador e a produtividade da empresa, o que se traduz em prejuízos físicos, psíquicos, econômicos e sociais. As consequências do álcool são diversas, a exemplo de redução no desempenho dos trabalhadores, altas taxas de rotatividade e elevação dos custos com benefícios de saúde (Barnes \& Zimmerman, 2013; Saade \& Marchand, 2013). 
O Consumo abusivo do álcool é agravado por sua associação com diversas consequências sociais. A Organização Pan-Americana de saúde (OPAS) aponta os efeitos negativos sobre a produtividade no trabalho, acidentes de trabalho e alto índice de absenteísmo (OPAS, 2019).

Por outro lado, as condições de trabalho da equipe de enfermagem, principalmente nos hospitais, têm sido consideradas impróprias, devido a remuneração inadequada, acumulação de escalas de serviço, aumento da jornada de trabalho, vivências constantes com o sofrimento humano e morte. Diante disso, esse cenário de trabalho torna-se um local gerador de riscos à saúde tanto físico quanto mental desses profissionais (Brotto \& Araujo, 2012).

Ainda nesse contexto, essas condições determinantes contribuem para o adoecimento desses profissionais, os quais procuram maneiras de fugir dessa realidade. Assim, o consumo de bebidas alcoólicas pode ser utilizado como uma maneira de lidar com o estresse, trazendo uma falsa sensação de relaxamento diante dos estressores vivenciados no ambiente de trabalho (Ferreira et al., 2013).

Ao analisar o uso de álcool no setor de saúde, em especial no cenário da enfermagem, percebe-se a gravidade diante das implicações negativas desse hábito, pois além dos impactos na vida do trabalhador, pode haver consequências e desfechos contrários na segurança dos cuidados prestados por esse grupo de profissionais (Voigt et al., 2009).

Esse consumo pode gerar condutas incorretas, o que se resume em cuidados ineficientes e risco potencial à segurança dos pacientes. Ademais, há risco adicional de ocorrência de acidente de trabalho para esses profissionais por déficit de concentração (Riboldi: Bordini \& Ferrario, 2012). Dessa forma o ensaio objetiva descrever os impactos do uso de bebidas alcoólicas por profissionais de enfermagem e a relação com o trabalho, a partir de uma reflexão teórica.

\section{Metodologia}

Trata-se de um ensaio teórico de caráter reflexivo, pautado na literatura cientifica sobre os impactos do uso de bebidas alcoólicas por profissionais de Enfermagem e a relação com o trabalho, por meio de uma revisão bibliográfica, com abordagem qualitativa, sobre o tema "uso de bebidas alcoólicas por profissionais de enfermagem e relação com o trabalho", e a partir da percepção dos autores sobre a temática. Corrobora Gil (2017) que a pesquisa bibliográfica permite analisar uma temática sob os olhares de diversos estudos de maneira abrangente e concisa.

A abordagem qualitativa nos ancora por responder questões muito particulares sem, contudo, preocupar-se com quantificações, dando ênfase ao fenômeno e às interpretações a ele atribuídas (Minayo, 2017).

Para a revisão bibliográfica utilizaram-se seis recursos eletrônicos, sendo cinco bases de dados eletrônicas: Literatura Latino-Americana e do Caribe em Ciências da Saúde (LILACS), Cummulative Index to Nursing and Allied Health Literature (CINAHL), Base de Dados de Enfermagem (BDENF), Web of Science - além do portal Pubmed, que engloba o Medical Literature Analysis and Retrievel System Online (MEDLINE), mantidos pela U.S. National Library of Medicine.

No portal Pubmed foram utilizados os descritores controlados do Medical Subject Headings (MESH) com a seguinte estratégia de busca: (Alcoholic Beverages OR Alcohol-Induced Disorders OR Alcohol-Related Disorders OR Alcohol Drinking) AND (Nurses OR Nurse Practitioners OR Nurse Midwives).

Nas bases Web of Science e CINAHL utilizou-se como estratégia de busca Alcohol AND Nurses. Nas bases de dados LILACS e BDENF, foram utilizados os Descritores em Ciências da Saúde - DeCs com a estratégia (Alcohol-Related Disorders OR Alcohol-Induced Disorders OR Alcohol Drinking) AND (Nurse Midwives OR Nurse Practitioners OR Nurses).

Foram incluídos os publicados entre os anos de 2010 a 2020, disponíveis na íntegra, na língua inglesa, portuguesa e espanhola, que traziam em seu conteúdo dados sobre consumo de bebidas alcoólicas por enfermeiros ou equipe de enfermagem. Foram excluídos artigos fora do recorte temporal, informes ou teses. A pesquisa foi realizada em maio de 2021. 
O processo de seleção dos estudos foi realizado seguindo as recomendações da metodologia PRISMA e os quantitativos de cada fase podem ser observados no fluxograma abaixo:

Figura 1 - Fluxograma PRISMA de seleção dos estudos.

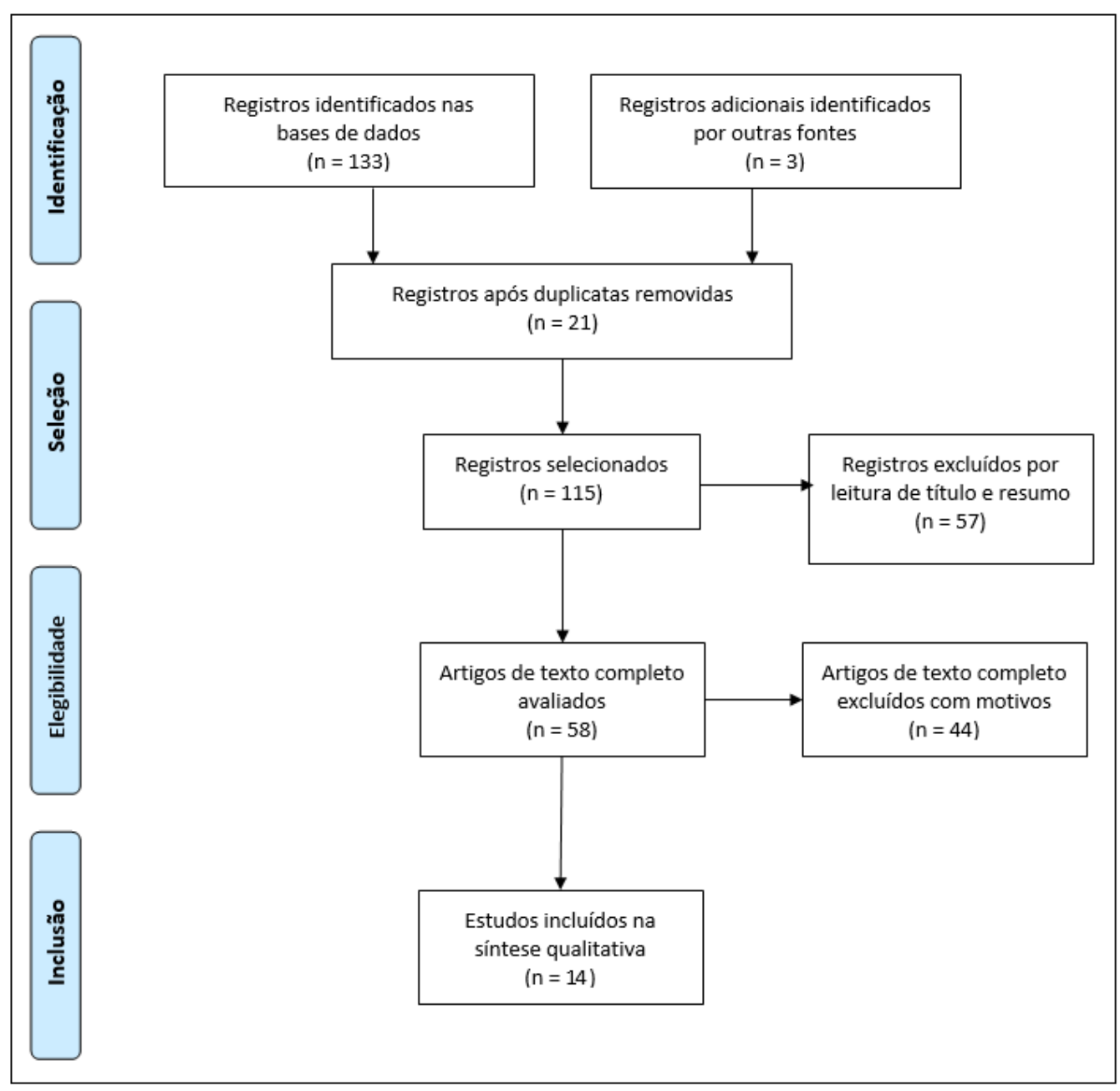

Fonte: Autores (2021).

Os artigos frutos das buscas foram objeto de construção de impressões e considerações da análise da literatura para reflexão e construção teórica acerca de dois subtemas.

\section{Resultados e Discussão}

A partir da leitura analítica e interpretativa dos artigos selecionados, foi realizada a divisão do conteúdo em duas unidades temáticas e as principais características dos 14 estudos selecionados estão descritas no quadro abaixo: 
Research, Society and Development, v. 10, n. 16, e61101623147, 2021

(CC BY 4.0) | ISSN 2525-3409 | DOI: http://dx.doi.org/10.33448/rsd-v10i16.23147

Quadro 1 - Características dos estudos selecionados.

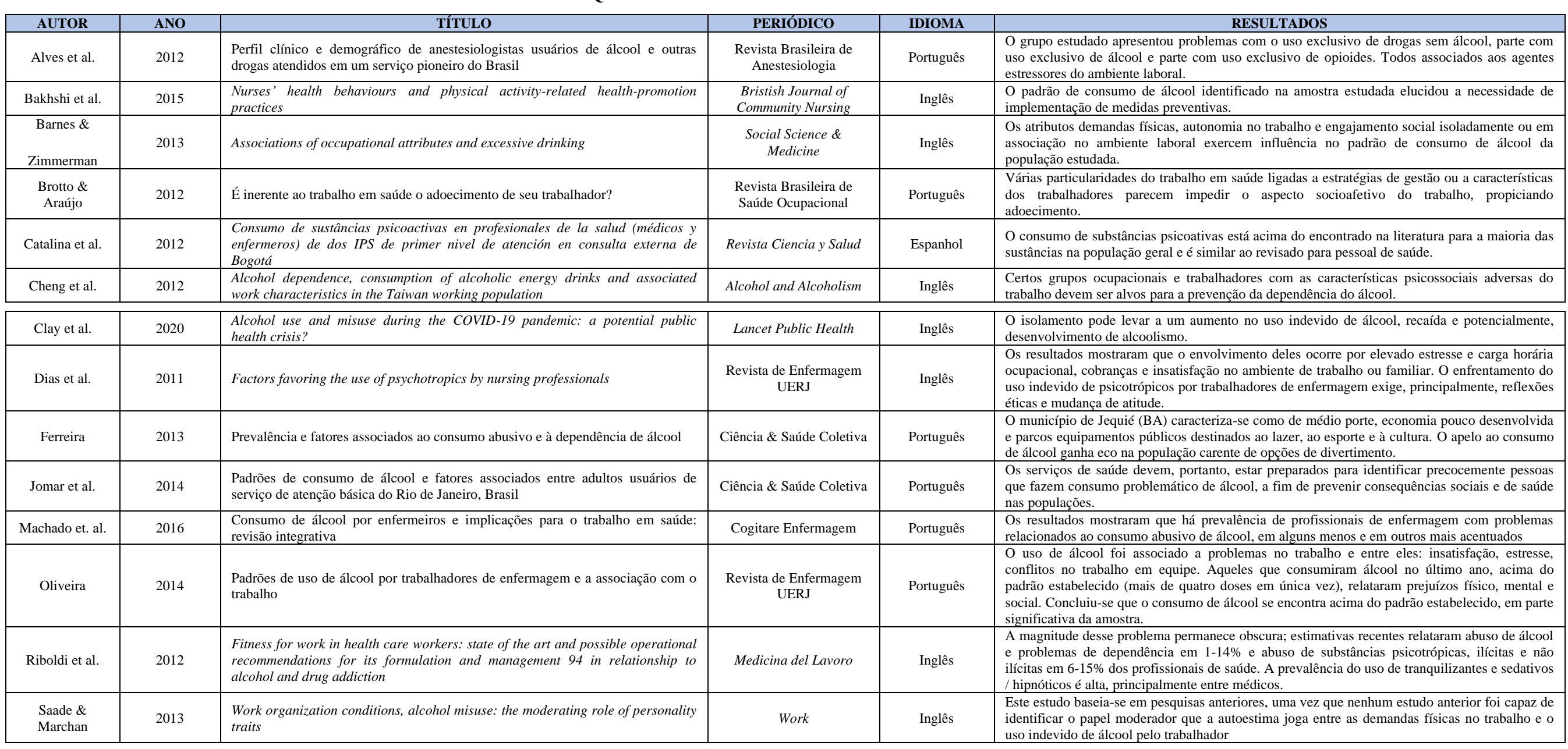

Fonte: Autores (2021). 


\section{Impactos do uso de bebidas alcoólicas por profissionais de Enfermagem}

Quanto aos impactos negativos do uso abusivo de álcool para o ambiente de trabalho, estatísticas da OIT (Organização Internacional do Trabalho) colocam o Brasil entre os cinco primeiros países do mundo em número de acidentes no trabalho, o que significa cerca de 500 mil acidentes por ano, sendo que quatro mil deles resultam em mortes (OPAS, 2020).

Ao analisarmos o cenário laboral onde está inserida a equipe de enfermagem percebemos que esses profissionais estão expostos fisicamente por exposição aos riscos de substâncias químicas, radiações, contaminações biológicas, jornada de trabalho excessiva; e psiquicamente em decorrência da convivência com o sofrimento e a dor, a doença e a morte, tendo ainda que lidar com suas questões emocionais. Essas condições podem favorecer ao uso de substâncias psicoativas, dentre elas, o álcool (Oliveira at. al., 2014).

Nesse contexto, as condições de trabalho adversas e os agentes estressores podem levar ao consumo excessivo de álcool, uma vez que os indivíduos encontram nessa substância uma alternativa de fuga da realidade e fonte de prazer e alegria, mesmo que momentâneo (Cheng et al., 2012).

Corrobora o estudo realizado por pesquisadores de Bogotá, na Colômbia que avaliou o uso de drogas lícitas entre médicos e enfermeiros, os resultados apontaram que o álcool, tabaco e bebidas energizantes são as mais prevalentes entre enfermeiros, apresentando maior consumo por mulheres, com uma média de 34 anos de idade para o abuso de álcool, 25 anos para o consumo de cigarro, e 24 a 49 anos para bebidas energizantes. Evidenciando o consumo de bebidas alcoólicas nessa população (Catalina \& Milena, 2012).

Um estudo realizado em hospital do Rio de Janeiro em 2014, cujo objetivo foi descrever padrões de consumo de álcool, com repercussão da equipe de enfermagem elucidou que 67\% dos participantes consumiram bebidas alcoólicas, nos últimos 12 meses. Além disso, 21,1\% dos entrevistados haviam ingerido, pelo menos, uma vez por semana no último ano. Destes, 6,6\% afirmaram consumir bebidas alcoólicas de duas a três vezes por semana. Quando inqueridos sobre os motivos que os levaram ao consumo, 26,6\% dos entrevistados associaram o uso à insatisfação no trabalho (Machado et al, 2016).

Há de se refletir que o fenômeno consumo de bebidas alcoólicas é um problema multidimensional, ocasionada não somente da mera relação existente entre uma pessoa e uma substância psicoativa, e sim da interação de ambos em um determinado contexto social, familiar e laboral que estabelece interconexões muitas vezes relacionadas ao estilo de vida e a forma como o sujeito lida com o espaço em que está inserido (Machado et al, 2016).

Embora o álcool possua um caráter lícito, essa substância é psicoativa e pode gerar dependência. Na perspectiva da relação entre consumo de álcool e trabalho, destaca-se que os problemas relacionados ao seu uso podem prejudicar a saúde do trabalhador e leva-lo à dependência (Jomar; Abreu \& Griep, 2014).

Por ser uma substância psicoativa, o álcool possui diversas ações no sistema nervoso central. Mesmo em doses baixas, apresenta efeitos sedativos, que causam relaxamento muscular e sonolência, bem como aumenta a liberação de dopamina, o que contribui para o efeito gratificante da droga. Além disso, gera desinibição social e déficits cognitivos temporários, o que resulta em dificuldades na resolução de problemas, alterações na memória e déficit de atenção (Andrade, 2020).

Neste sentido, o consumo de bebidas alcoólicas pela equipe de enfermagem e a associação com o mundo do trabalho traz como consequência implicações negativas desse hábito na vida do trabalhador e, sobretudo, gerar consequências e desfechos na segurança de quem recebe os cuidados prestados pela enfermagem (Voigt et al., 2009).

\section{Fatores associados ao consumo de bebidas alcoólicas no ambiente de trabalho da enfermagem}

É possível que o ritmo acelerado de vida e no ambiente laboral, exige cada vez mais dos profissionais, e consequentemente -estimula a competitividade, diante das novas relações de trabalho, além de carga horária excessiva, 
trabalho noturno, ambiente laboral de grande estresse e que exige constante vigilância. Diante disso, podem influenciar no consumo de bebidas alcoólicas pelo trabalhador (Alves et al., 2012).

Sendo assim, os profissionais de enfermagem estão sob pressão constante relacionada ao trabalho, além de estarem num ambiente insalubre, vivenciando situações de estresse relacionado às situações emergenciais e plantões noturnos. Adicionalmente, o consumo de álcool pode ser um mecanismo de enfrentamento para lidar com a insônia, ansiedade e dor física (Alves et al., 2012).

Outro ponto a ser considerado é a crise sanitária que estamos atravessando caracterizada pela atuação incessante da equipe de enfermagem na linha de frente contra a Covid-19, os levando ao esgotamento físico e mental.

O estudo realizado pela FIOCRUZ em março deste ano para avaliar o impacto da pandemia de Covid-19 entre os profissionais de saúde apontou que as alterações mais comuns em seu cotidiano, citadas pelos profissionais, foram perturbação do sono (15,8\%), irritabilidade/choro e frequente/distúrbios em geral (13,6\%), incapacidade de relaxar/estresse (11,7\%), dificuldade de concentração ou pensamento lento $(9,2 \%)$, perda de satisfação na carreira ou na vida/tristeza/apatia $(9,1 \%)$, sensação negativa do futuro/pensamento negativo, suicida $(8,3 \%)$ e alteração no apetite/alteração do peso $(8,1 \%)$ ( Leonel, 2021).

Deste modo, o ambiente laboral e todo o contexto que o envolve corresponde como um facilitador para o consumo de bebidas alcoólicas por trabalhadores de enfermagem, haja vista a pré-disposição do sujeito, além de suas crenças, valores, condição sociodemográfica e a própria relação com o trabalho (Dias et. al., 2011).

Considerando que, fora do ambiente de trabalho esses profissionais também vivenciam o isolamento social. Os efeitos do isolamento e o consumo de álcool ainda não estão bem estabelecidos nos diferentes países (Clay \& Parker, 2020).

Importa mencionar que o estresse é um fator de risco crucial para o início e manutenção do uso de bebidas alcoólicas. Assim, por se tratar de um evento estressor e altamente ansiogênico, as medidas de restrição de contato interpessoal e isolamento social podem acarretar sobrecarga psicológica que incrementa o uso indevido de álcool, podendo ocasionar o pico de consumo facilmente, principalmente em indivíduos em maior risco de vulnerabilidade psicossocial (Clay \& Parker, 2020).

\section{Conclusão}

O ensaio teórico possibilitou identificar nos estudos que o consumo de bebidas alcoólicas pela equipe de enfermagem é uma realidade no cenário laboral dessa categoria profissional e apresenta interfaces com a realidade presente no cotidiano desses trabalhadores, e a estreita relação com as condições laborais em que desempenham suas atividades.

Os estudos analisados permitiram compreender que dentre os impactos do uso de bebidas alcoólicas por profissionais de enfermagem, destacam- se os danos que repercutem diretamente na qualidade da assistência, comprometendo não só a segurança do paciente, mas ocasionando uma problematização que afeta todo o sistema institucional de saúde, podendo trazer, ainda, complicações para à saúde, bem como na vida pessoal, social e profissional desses indivíduos. Os erros assistências, absenteísmo, dependência química e perda do registro de classe.

Com relação aos fatores associados ao consumo de bebidas alcoólicas no ambiente de trabalho da enfermagem, houve destaque para os fatores estressores, para a crise sanitária atual em decorrência da Covid-19 e do isolamento social.

É necessário que a partir dessa reflexão possamos pensar em estratégias para o fortalecimento de ações de promoção e educação em saúde, voltadas para a melhoria da saúde do trabalhador.

E por fim, chama-se a atenção para a necessidade da realização de novos estudos de cunho metodológicos mais complexos com o intuito de investigar a questão do uso de bebidas alcoólicas no cenário saúde. 


\section{Referências}

Alves, H. N. P., Vieira, D. L., Laranjeira, R. R., Vieira, J. E. \& Martins, L. A. N. (2012). Perfil clínico e demográfico de anestesiologistas usuários de álcool e outras drogas atendidos em um serviço pioneiro do Brasil. Revista Brasileira de Anestesiologia, 62(3), 356-364.

Andrade, A. G. (2020). Álcool e a Saúde dos Brasileiros: Panorama 2020. CISA.

Bakhshi, S., Sun, F., Murrells, T. \& While, A. (2015). Nurses' health behaviours and physical activity-related health-promotion practices. British Journal of Community Nursing, 20(6), 289-96.

Barnes, A. J. \& Zimmerman, F. J. (2013). Associations of occupational attributes and excessive drinking. Social Science \& Medicine, 92 , 35-42.

Brotto, T. C. A. \& Araujo, M. D. (2012). É inerente ao trabalho em saúde o adoecimento de seu trabalhador? Revista Brasileira de Saúde Ocupacional, 37(126), 290-305.

Catalina, L. H., Milena, V. C. G., Alejandra, S. M. (2012). Consumo de sustancias psicoactivas en profesionales de la salud (médicos y enfermeros) de dos IPS de primer nivel de atención en consulta externa de Bogotá. Revista Ciencia y Salud, 10(Suppl 1), 87-100.

Cheng, W. J., Cheng, Y., Huang, M. C. \& Chen, C. J. (2012). Alcohol dependence, consumption of alcoholic energy drinks and associated work characteristics in the Taiwan working population. Alcohol and Alcoholism, 47(4), 372-379.

Clay, J. M. \& Parker, M. O. (2020). Alcohol use and misuse during the COVID-19 pandemic: a potential public health crisis? Lancet Public Health, 5(5), e259.

Dias, J. R. F., Araújo, C. S., Martins, E. R. C., Clos, A. C., Francisco, M. T. R. \& Sampaio, C. E. P. (2011). Factors favoring the use of psychotropics by nursing professionals. Revista de Enfermagem UERJ, 19(3), 445-451.

Ferreira, L. N., Bispo JR, J. P., Sales, Z. N., Casotti, C. A. \& Braga Jr, A. C. R. (2013). Prevalência e fatores associados ao consumo abusivo e à dependência de álcool. Ciência e Saúde Coletiva, 18(11), 3409-3418.

Gil, A. C. (2017). Como elaborar projetos de pesquisa. (6a ed.), Atlas.

Jomar, R. T., Abreu, A. M. \& Griep, R. H. (2014). Padrões de consumo de álcool e fatores associados entre adultos usuários de serviço de atenção básica do Rio de Janeiro, Brasil. Ciência \& Saúde Coletiva, 19(1), 27-37.

Leonel, F. (2021). Pesquisa analisa o impacto da pandemia entre profissionais de saúde. FIOCRUZ. https://portal.fiocruz.br/noticia/pesquisa-analisa-oimpacto-da-pandemia-entre-profissionais-de-saude.

Machado, A. S., Monteiro, P. S., Ribeiro, L. M. \& Guilhem, D. (2016). Consumo de álcool por enfermeiros e implicações para o trabalho em saúde: revisão integrativa. Cogitare Enfermagem, 21(4), 01-08.

Minayo, M. C. S. (2017). Amostragem e saturação em pesquisa qualitativa: consensos e controvérsias. Revista Pesquisa Qualitativa, 5(7), 1-12.

Oliveira, E. B., Fabri, J. M. G., Paula, G. S., Souza, S. R. C., Silveira, W. G. \& Matos, G. S. (2014). Padrões de uso de álcool por trabalhadores de enfermagem e a associação com o trabalho. Revista de Enfermagem UERJ, 21(6), 729-735.

Organização Pan-Americana da Saúde. (2019). Folha informativa: álcool. https://www.paho.org/bra/index.php?option=com_content\&view=arti cle\&id=5649: folha-informativa-alcool\&Itemid=1093.

Pan American Health Organization. (2020). Regional Status Report on Alcohol and Health in the Americas 2020. PAHO.

Riboldi, L., Bordini, L. \& Ferrario, M. M. (2012). Fitness for work in health care workers: state of the art and possible operational recommendations for its formulation and management 94 in relationship to alcohol and drug addiction. Medicina del Lavoro, 103(3), 203-2011.

Saade, S. L. \& Marchand, A. (2013). Work organization conditions, alcohol misuse: the moderating role of personality traits. Work, 44(2), 191-200.

Voigt, K., Twork, S., Mittag, D., Göbel, A., Voigt, R., Klewer, J., Kugler, J., Bornstein, S. R. \& Bergmann, A. (2009). Consumption of alcohol, cigarettes and illegal substances among physicians and medical students in Brandenburg and Saxony (Germany). BMC Health Services Research, 9(219), 1-7.

World Health Organization. (2018). Global Status Report on Alcohol and Health 2018. WHO. 